\title{
Extremely weak CO emission in IZw 18
}

\author{
Luwenjia Zhou (周陆文嘉) $)^{1,2} \odot$, Yong Shi (施勇 $)^{1,2}$, Zhi-Yu Zhang (张智昱) $)^{1,2}$, and Junzhi Wang (王均智 $)^{3}$ \\ ${ }^{1}$ School of Astronomy and Space Science, Nanjing University, Nanjing 210093, PR China \\ e-mail: wenjia@nju.edu.cn, yshipku@gmail.com \\ 2 Key Laboratory of Modern Astronomy and Astrophysics (Nanjing University), Ministry of Education, Nanjing 210093, PR China \\ 3 Shanghai Astronomical Observatory, Chinese Academy of Sciences, 80 Nandan Road, Shanghai 200030, PR China
}

Received 26 July 2020 / Accepted 9 September 2021

\begin{abstract}
Local metal-poor galaxies are ideal analogues of primordial galaxies with the interstellar medium (ISM) barely being enriched with metals. However, it is unclear whether carbon monoxide remains a good tracer and coolant of molecular gas at low metallicity. Based on the observation with the upgraded Northern Extended Millimeter Array, we report a marginal detection of CO $J=2-1$ emission in IZw18, pushing the detection limit down to $L_{\mathrm{CO}(2-1)}^{\prime}=3.99 \times 10^{3} \mathrm{~K} \mathrm{~km} \mathrm{~s}^{-1} \mathrm{pc}^{-2}$, which is at least 40 times lower than previous studies. As one of the most metal-poor galaxies, IZw18 shows extremely low CO content despite its vigorous star formation activity. Such low CO content relative to its infrared luminosity, star formation rate, and [C II] luminosity, compared with other galaxies, indicates a significant change in the ISM properties at a few percent of the Solar metallicity. In particular, the high [C II] luminosity relative to CO implies a larger molecular reservoir than the CO emitter in IZw18. We also obtain an upper limit of the 1.3 mm continuum, which excludes a sub-millimetre excess in IZw18.
\end{abstract}

Key words. galaxies: dwarf - ISM: molecules

\section{Introduction}

Star formation occurs in molecular gas dominated by $\mathrm{H}_{2}$, except perhaps for the star formation in the early universe. However, $\mathrm{H}_{2}$ can only be excited at a temperature above $100 \mathrm{~K}$, hence it cannot be observed directly in the cold molecular gas that fuels star formation. The second most abundant molecule, $\mathrm{CO}$, has been demonstrated to be a powerful tracer of molecular clouds in galaxies, but this application becomes complicated in metalpoor environments (see Bolatto et al. 2013, for a review).

In the early Universe, the first galaxies formed in the primordial gas with few elements being heavier than hydrogen. Observationally, at $z \gtrsim 5$, normal star-forming galaxies with star formation rates (SFRs) of ten to a few hundred solar masses per year indeed have a low dust content (Walter et al. 2012; Capak et al. 2015), comparable to local metal-poor galaxies. Nevertheless, the predicted CO flux in these high- $z$ galaxies is of the order of $\mu \mathrm{Jy}$ and remains challenging due to the state-of-the-art sub-millimetre array, Atacama Large Millimeter/submillimeter Array (ALMA). A detailed study on the metalpoor interstellar medium (ISM) relies on its local analogues.

Despite the extensive search for $\mathrm{CO}$ emissions in local dwarf galaxies (Leroy et al. 2007; Schruba et al. 2012; Cormier et al. 2014; Hunt et al. 2015; Warren et al. 2015; Shi et al. 2015), the detection rate decreases sharply in galaxies with a metallicity lower than one-fifth the Solar metallicity $\left(Z_{\odot}\right)^{1}$. The extremely faint $\mathrm{CO}$ emission brings about questions as to the existence of molecular gas in such galaxies. On the other hand, CO may not be an ideal tracer of molecular gas (Grenier et al. 2005;

\footnotetext{
1 Here we adopt Solar metallicity as $12+\log (\mathrm{O} / \mathrm{H})=8.7$ (Asplund et al. 2009).
}

Wolfire et al. 2010; Shi et al. 2016) in low-metallicity environments. The interstellar radiation penetrates deeper into molecular clouds due to the low dust content. At such depths within the clouds, $\mathrm{CO}$ is more easily destroyed than $\mathrm{H}_{2}$ through dissociation, and thus it ceases to be a reliable tracer. In addition, the reduced opacity in the stellar atmosphere leads to harder radiation fields, and hence reinforces the destruction of $\mathrm{CO}$ molecules into atomic or singly ionised carbon.

Low CO content is then expected in metal-poor galaxies. On the other hand, exposed to the hard radiation field, $\mathrm{CO}$ molecules can be easily photodissociated and produce ionised carbon, [C II]. Furthermore, [C II] could come from ionised gas as well as neutral gas and the surface of photodissociation regions (PDRs). However, the fractions of [C II] emission from ionised gas derived from observation constraints are systematically lower than the one from simulations (Accurso et al. 2017b; Cormier et al. 2019). Therefore it is still unclear whether it is feasible to use [C II] to trace $\mathrm{H}_{2}$ gas.

Substantial efforts have been made to search for the molecular gas in metal-poor galaxies (Rubio et al. 2015; Oey et al. 2017; Schruba et al. 2017; Elmegreen et al. 2018); nevertheless, among the galaxies below $10 \% Z_{\odot}$, only Sextans B $\left(7 \% Z_{\odot}\right)$ has a robust CO detection (Shi et al. 2016, 2020). The impact of low metal abundance on star formation requires further exploration of the most metal-poor galaxies. In this work, we used the upgraded NOEMA interferometer to observe the CO $J=2-1$ emission in IZw18, which is one of the galaxies with the lowest metallicities in the local Universe $\left(\sim 3 \% Z_{\odot}\right.$, Izotov \& Thuan 1999). IZw18 is a proto-type of blue compact dwarfs located at a distance of $18.2 \mathrm{Mpc}$ (Aloisi et al. 2007). The active star formation therein (Hunt et al. 2005) indicates the presence of molecular gas. Leroy et al. (2007) obtained an upper limit of 
Table 1. Observation information.

\begin{tabular}{|c|c|c|c|c|c|c|c|}
\hline & \multirow[t]{2}{*}{ \# of Antennas } & \multicolumn{2}{|l|}{ Calibrators } & \multirow[t]{2}{*}{$\mathrm{PWV}^{(1)}$} & \multirow[t]{2}{*}{$T_{\text {sys }}$} & \multicolumn{2}{|c|}{ Resulting sensitivity } \\
\hline & & Phase/Amplitude & Bandpass & & & Line $^{(2)}$ & Continuum ${ }^{(3)}$ \\
\hline obs-17 & 9 & $0954+556,1030+611$ & $3 \mathrm{C} 273$ & $3 \mathrm{~mm}$ & $\sim 156.1 \mathrm{~K}$ & $1.5 \mathrm{mJy}$ beam $^{-1}$ & $28.9 \mu \mathrm{Jy}_{\text {beam }^{-1}}$ \\
\hline $\begin{array}{l}\text { obs-19 } \\
\text { merged }\end{array}$ & 9 & $0925+504,0954+556$ & $3 \mathrm{C} 84$ & $3 \mathrm{~mm}$ & $\sim 178.1 \mathrm{~K}$ & $\begin{array}{l}1.8 \mathrm{mJy}_{\text {beam }}^{-1} \\
1.3 \mathrm{mJy} \mathrm{beam}^{-1}\end{array}$ & $\begin{array}{l}34.5 \mu \mathrm{Jy}_{\text {beam }}-1 \\
26.2 \mu \mathrm{Jy}_{\text {beam }}-1\end{array}$ \\
\hline
\end{tabular}

Notes. ${ }^{(1)}$ Precipitable water vapour. ${ }^{(2)}$ For the $3 \mathrm{D}$ cubes smoothed to $3 \mathrm{~km} \mathrm{~s}^{-1}$ and reconstructed with natural weighting. ${ }^{(3)}$ For the images tapered to a beam size of $2^{\prime \prime}$.

$\mathrm{CO}(1-0)$ emission $\left(L_{\mathrm{CO}}^{\prime}<10^{5} \mathrm{~K} \mathrm{~km} \mathrm{~s}^{-1} \mathrm{pc}^{2}\right)$ using the Plateau de Bure Interferometer (PdBI), which however is not deep enough to conclude whether IZw 18 has a normal CO content relative to other physical properties. Here we put further constraints on the CO content in IZw18 by pushing down the detection limit of $L_{\text {CO }}^{\prime}$ by 40 times using the Northern Extended Millimeter Array (NOEMA) after its Phase II upgrade.

\section{Observations}

The observations of IZw18 were carried out with NOEMA on December 24, 2017 (obs-17) and March 23, 2019 (obs-19) for a total of $16 \mathrm{~h}$ ( $11.6 \mathrm{~h}$ on source) with configuration D. We processed the data at IRAM/Grenoble using the GILDAS package. The final CO $J=2-1$ datacubes of obs17, obs18, and the merger of the two have beam sizes of 1 '.83 81 1"60 (obs-17), $1^{\prime \prime} .71 \times 11^{\prime \prime} .41$ (obs-18), and 1 '.71 $\times 11^{\prime \prime} .48$ (merged), respectively, and a frequency resolution of $0.2 \mathrm{MHz}\left(0.26 \mathrm{~km} \mathrm{~s}^{-1}\right.$ at $\left.230 \mathrm{GHz}\right)$. IZw18 was entirely covered by the $22^{\prime \prime}$ (FWHM) primary beam. The details of the observation settings and the sensitivities of the data are listed in Table 1.

\section{Results}

\section{1. $C O J=2-1$}

We smoothed the data to a velocity resolution of $3 \mathrm{~km} \mathrm{~s}^{-1}$, consistent with the $\mathrm{CO}$ line width found in the most metalpoor galaxies in the literature (Shi et al. 2016). We found one marginal detection $\left(\alpha=9^{\mathrm{h}} 34^{\mathrm{m}} 02^{\mathrm{s}} 00, \delta=55^{\circ} 14^{\prime} 28^{\prime \prime} .81\right)$ at the peak of the stellar emission, as indicated by the red contour ${ }^{2}$ superimposed on the Hubble Space Telescope (HST) $V$-band image (Fig. 1) ${ }^{3}$. It covers an area defined by the $3 \sigma$ contour of $\sim 1^{\prime \prime} .52 \times 11^{\prime \prime} .52$, which is approximately the size of the synthesised beam. We have plotted the spectra from the merged, obs17 and obs-19 data cubes in Fig. 2. A $3 \sigma$ spectral signal falls at $46 \mathrm{~km} \mathrm{~s}^{-1}$. The peak fluxes derived from the peak channels have signal-to-noise ratios of $3.47,2.73$, and 3.45 for the merged, obs17 and obs-19 data cubes. We note that even though the spectrum extracted from the obs-17 data cube shows lower rms noise, the noise level in the data cube slightly deviates from a Gaussian distribution, while those from obs-19 and merged data cubes do not. Hereafter, we use the result from the merged data cube.

The CO $J=2-1$ peak flux density is $S_{\mathrm{CO}(2-1)}=2.48 \mathrm{mJy}$ and the corresponding $\mathrm{CO}$ luminosity is then derived to be $L_{\mathrm{CO}(2-1)}^{\prime}=3.99 \times 10^{3} \mathrm{~K} \mathrm{~km} \mathrm{~s}^{-1} \mathrm{pc}^{-2}$ using the formulation of

\footnotetext{
2 The red contours show the $\mathrm{CO} J=2-1$ line intensity integrated from $40 \mathrm{~km} \mathrm{~s}^{-1}$ to $50 \mathrm{~km} \mathrm{~s}^{-1}$, where the marginal detection falls, as shown in Fig. 2.

3 We determined the HST astrometry in this field to be flawed by $\sim 0.3^{\prime \prime}$, and hence negligible.
}

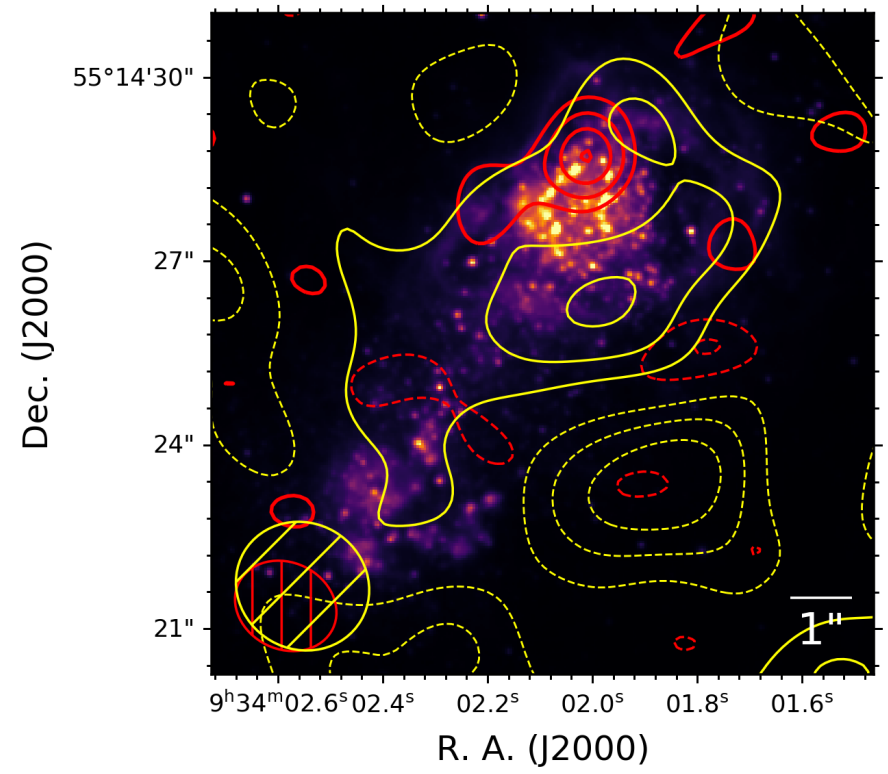

Fig. 1. CO $J=2-1$ emission and $1.3 \mathrm{~mm}$ continuum superimposed on the HST $V$-band image from Izotov \& Thuan (2004, PropID: 9400). The red contours denote the CO $J=2-1$ integrated line intensity at the resolution of 1 '.71 $71^{\prime \prime} .48$, starting from $2 \sigma$, in increments of $1 \sigma\left(3.3 \mathrm{mJy}\right.$ beam $\left.^{-1}\right)$ significance. The yellow contours denote the continuum detection at $1.3 \mathrm{~mm}$ at the resolution of $2^{\prime \prime} .21 \times 2^{\prime \prime} .06$, starting from $1 \sigma$, in increments of $1 \sigma\left(24 \mu \mathrm{Jy}_{\text {beam }}{ }^{-1}\right)$ significance. Dashed contours are negative.

Solomon \& Vanden Bout (2005). The CO emission tends to reside in clumps of a few parsecs at low metallicity (Rubio et al. 2015; Oey et al. 2017; Schruba et al. 2017; Shi et al. 2020), which is much smaller than the beam size of our observation, hence we do not expect significant missing diffuse emission.

\section{2. $1.3 \mathrm{~mm}$ continuum}

We created a continuum map after removing channels with emission. The continuum map was smoothed to have an angular resolution of 2 ". $21 \times 2$..06 with an rms noise of $51 \mu \mathrm{Jy} \mathrm{beam}^{-1}$, using the TCLEAN TASK IN CASA. The flux peak falls at $\alpha=9^{\mathrm{h}} 34^{\mathrm{m}} 02^{\mathrm{s}} 00, \delta=55^{\circ} 14^{\prime} 26^{\prime \prime}$ ' 30 which is between the two flux peaks of the H I emission of IZw18 (Lelli et al. 2012, see also Appendix A). This is the only $3 \sigma$ signal $\left(F_{1.3 \mathrm{~mm}}=181 \mu \mathrm{Jy}\right)$ within the primary beam. Comparisons between CO $J=2-1$ emission and $1.3 \mathrm{~mm}$ continuum as well as the emission at FUV $, 3.6 \mu \mathrm{m}, 24 \mu \mathrm{m}, 100 \mu \mathrm{m}$, and of $\mathrm{HI}$ gas are shown in Appendix A. 


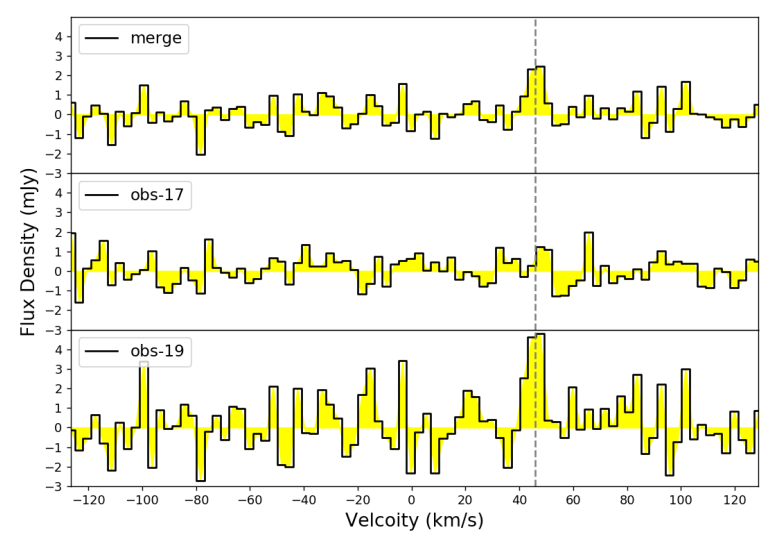

Fig. 2. CO $J=2-1$ spectra from the merged, obs-17 and obs-19 data cubes. The spectra are smoothed to a velocity resolution of $3 \mathrm{~km} \mathrm{~s}^{-1}$ and extracted from the area defined by the $3 \sigma$ contour as shown in Fig. 1 . The $1 \sigma$ uncertainty is $0.72,0.72$, and $1.39 \mathrm{mJy}$, respectively. The grey dashed line denotes the marginal detection at $\sim 46 \mathrm{~km} \mathrm{~s}^{-1}$.

Table 2. Basic information on IZw18 used in this paper.

\begin{tabular}{lcc}
\hline \hline Parameter & Value & Reference \\
\hline$V_{\text {hel }}$ & $751 \mathrm{~km} \mathrm{~s}^{-1}$ & $(1)$ \\
$D$ & $18.2 \mathrm{Mpc}$ & $(2)$ \\
$12+\log (\mathrm{O} / \mathrm{H})$ & 7.17 & $(3)$ \\
$M_{\star}$ & $4.33 \times 10^{6} M_{\odot}$ & This paper. $^{(a)}$ \\
$S F R$ & $0.12 \pm 0.01 \mathrm{MJ}_{\odot} \mathrm{yr}^{-1}$ & This paper. $^{(b)}$ \\
$S_{\mathrm{CO}(2-1)}$ & $2.48 \mathrm{mJy}^{(c)}$ & This paper. $^{(d)}$ \\
$F_{\mathrm{CO}(2-1)}$ & $19.42 \mathrm{mJy} \mathrm{km} \mathrm{s}^{-1}$ & This paper. $^{(d)}$ \\
$L_{\mathrm{CO}(2-1)}^{\prime}$ & $3.99 \times 10^{3} \mathrm{~K} \mathrm{~km} \mathrm{~s}^{-1} \mathrm{pc}^{2}$ & This paper. $^{(e)}$ \\
$L_{\mathrm{CO}(1-0)}$ & $0.18 \mathrm{~L}_{\odot}$ & This paper. $^{(e)}$ \\
$F_{1.3 \mathrm{~mm}}$ & $181 \pm 51 \mu \mathrm{Jy}$ & This paper. $^{(f)}$ \\
\hline
\end{tabular}

Notes. ${ }^{(1)}$ NASA/IPAC Extragalaxtic Database (NED). ${ }^{(2)}$ Aloisi et al. (2007). ${ }^{(3)}$ Main body in the south-west (Izotov \& Thuan 1999). ${ }^{(a)}$ Derived from the SED fitting in Sect. 4.1. ${ }^{(b)}$ Based on the emission at FUV and $24 \mu \mathrm{m} .{ }^{\left({ }^{c}\right)}$ Peak flux density of $\mathrm{CO}(2-1)$ as described in Sect. 3.1. ${ }^{(d)}$ Velocity integrated line flux of $\mathrm{CO}(2-1)$ from $40 \mathrm{~km} \mathrm{~s}^{-1}$ to $50 \mathrm{~km} \mathrm{~s}^{-1} \cdot{ }^{(e)} \mathrm{CO}(1-0)$ luminosity. Assuming optically thick and thermalised $L_{\mathrm{CO}(1-0)}^{\prime}=L_{\mathrm{CO}(2-1)}^{\prime}{ }^{(f)} 1.3 \mathrm{~mm}$ continuum flux as described in Sect. 3.2.

\section{Discussion}

In this section, we discuss how the CO luminosity and the $1.3 \mathrm{~mm}$ continuum flux constrain our current understanding of the star formation process in the extremely metal-poor environment in IZw18. The physical parameters we used for IZw18 are listed in Table 2.

We compare IZw18 to normal star-formimg galaxies and metal-poor galaxies in the literature. These include seven metalpoor dwarf galaxies from the Herschel Dwarf Galaxy Survey (DGS, Cormier et al. 2014, compiled in Accurso et al. 2017b), 16 nearby dwarf galaxies in Schruba et al. (2012) and nearby galaxies compiled therein, eight metal-poor galaxies from Hunt et al. (2015), local galaxies with stellar mass higher than $10^{9} M_{\odot}$ from the xCOLD GASS survey (Saintonge et al. 2017), and massive, infrared bright, local star-forming galaxies at Solar metallicity from Gao \& Solomon (2004), as well as the individual star-forming regions in four extremely metal-poor galaxies $(12+\log (\mathrm{O} / \mathrm{H})<8)$ from Shi et al. $(2015,2016)$.

\subsection{SED and sub-millimetre excess}

We first investigated the spectral energy distribution (SED) of IZw18 taking into account the $3 \sigma$ upper limit at $1.3 \mathrm{~mm}$ to put more constraints on the FIR/millimetre regime. All other photometric data were taken from Hunt et al. (2014). We performed the SED fitting with CIGALE (Boquien et al. 2019) adopting a delayed star formation history, a Draine \& Li (2007) dust model, which is consistent with the method used for the star-forming regions in Shi et al. $(2015,2016)$, and a radio component. The infrared luminosity derived from the fit is $2.2 \times 10^{7} L_{\odot}$, consistent with the one from Hunt et al. (2014). The stellar mass is $4.33 \times 10^{6} M_{\odot}$, within the uncertainty of the stellar masses derived from stellar mass-to-light ratios in the $K$ band and $3.6 \mu \mathrm{m}$ (Fumagalli et al. 2010; Hunt et al. 2019), while it is around ten times lower than those derived based on the $R$ band luminosity (Lelli et al. 2012, 2014).

We also explored the existence of the excess at sub-millimeter wavelengths (e.g., Galliano et al. 2003; Lisenfeld et al. 2002; Galametz et al. 2011; Rémy-Ruyer et al. 2013) in IZw18. It did not have a robust detection at the far-infrared (FIR) wavelengths longer than $160 \mu \mathrm{m}$. The $3 \sigma$ upper limit at $1.3 \mathrm{~mm}$ places a strong constraint on the (sub-) millimetre end of the dust emission. We fitted the FIR SED with the modified blackbody model and derived a lower limit of the dust emissivity $\beta$ to be 2.1 , which suggests no sub-millimetre excess in IZw18. Furthermore, taking into account the upper limit at $1.3 \mathrm{~mm}$, we notice that the radio emission shows a steeper slope $(\alpha=-0.58)$, compared to the spectral indice found in Hunt et al. (2005) $\left(\alpha_{1.4 \mathrm{GHz}}^{4.8 \mathrm{GHz}}=-0.39, \alpha_{4.8 \mathrm{GHz}}^{8.4 \mathrm{GHz}}=-0.13\right)$; this may be due to the high frequency cutoff of synchrotron emission from the relavitistic electrons in the star-forming regions. Therefore, we fitted the radio emission with a cutoff model as introduced in Klein et al. (2018), by fixing the spectral index of the synchrotron emission to be $\alpha_{n \text {th }}=\alpha_{1.4 \mathrm{GHz}}^{4.8 \mathrm{GHz}}=-0.39$ (Fig. 3). Then the contribution of the free-free emission to the total radio emission is $f_{1.3 \mathrm{~mm}}^{\mathrm{ff}} \sim 76 \%$ and $f_{2.6 \mathrm{~mm}}^{\mathrm{ff}} \sim 54 \%$, higher than what was found in the literature (e.g. Leroy et al. 2007; Hunt et al. 2014). We note that the cutoff frequency derived from the fit is at $145 \mathrm{GHz}$, which is higher than the typical value of $\sim 10 \mathrm{GHz}$ found in Klein et al. (2018).

\section{2. $L_{\mathrm{IR}}$ and $S F R$ versus $L_{\mathrm{CO}}^{\prime}$}

In Fig. 4, we have plotted the total infrared luminosity (8$1000 \mu \mathrm{m}), L_{\mathrm{IR}}$, versus $L_{\mathrm{CO}(1-0)}^{\prime}$ and the SFR versus $L_{\mathrm{CO}(1-0)}^{\prime}$. These two correlations have been well established in massive star-forming galaxies (e.g. Gao \& Solomon 2004) as CO molecules and $L_{\mathrm{IR}}$ trace $\mathrm{H}_{2}$ molecular clouds and dust emission, respectively. We assume optically thick and thermalised $\mathrm{CO}$ emission, then $L_{\mathrm{CO}(1-0)}^{\prime}=L_{\mathrm{CO}(2-1)}^{\prime}$. We note that $\mathrm{CO}$ tends to become warm and optically thin as $\mathrm{CO}$ is exposed to interstellar radiation in metal-poor galaxies. Schruba et al. (2012) adopted $R_{21}=0.7$, which is the average value of HERACLES galaxies (Rosolowsky et al. 2015) for nearby dwarf galaxies. Saintonge et al. (2011) studied the 523 galaxies in xCOLD GASS survey and gave an average value of $0.79 \pm 0.03$ with a larger scatter at $L_{\mathrm{CO}(1-0)}^{\prime}<10^{8} \mathrm{~K} \mathrm{~km} \mathrm{~s}^{-1} \mathrm{pc}^{2}$. Therefore, the choice of $R_{21}$ may lead to the derived $L_{\mathrm{CO}(1-0)}^{\prime}$ a factor of 1.4 different from the one based on our assumption. The SFR was derived from the combination of FUV and $24 \mu \mathrm{m}$ emission for all galaxies, except for the infrared bright galaxies for which the SFR was derived from infrared luminosity. 


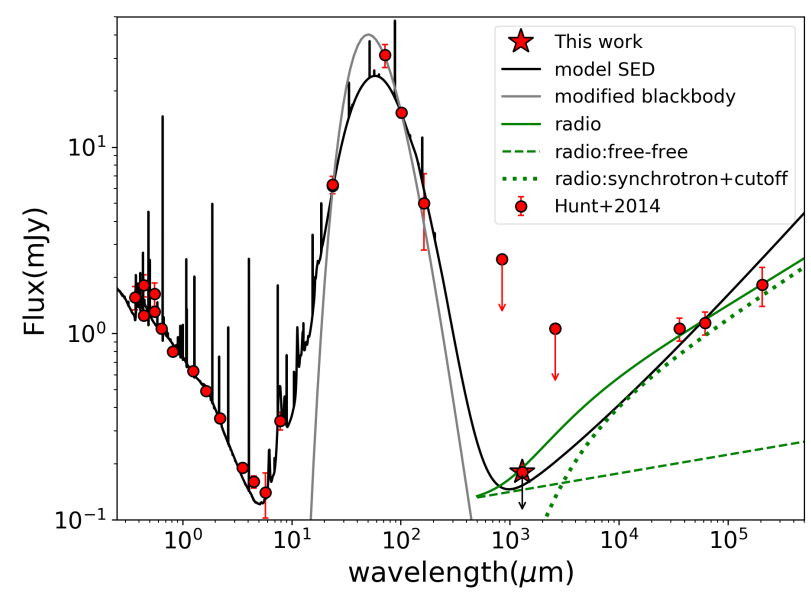

Fig. 3. SED of IZw18, with CIGALE best fit adopting a delayed star formation history, a Draine \& $\mathrm{Li}$ (2007) dust model, and a radio component. A modified blackbody fit is shown with a grey line. Photometric data are from Hunt et al. (2014), except for at $1.3 \mathrm{~mm}$. The $3 \sigma$ upper limit at $1.3 \mathrm{~mm}$ is shown as in the red star. The radio emission is also fitted with a cutoff model including a free-free component and a synchrotron component as introduced in Klein et al. (2018) (see more in the Sect. 4.1), which is indicated by the green curves.

Compared to star-forming galaxies with Solar metallicity, metal-poor galaxies show higher $L_{\mathrm{IR}} / L_{\mathrm{CO}(1-0)}^{\prime}$ and $\mathrm{SFR} / L_{\mathrm{CO}(1-0)}^{\prime}$ ratios. The $L_{\mathrm{IR}} / L_{\mathrm{CO}(1-0)}^{\prime}$ ratio of metal-poor galaxies is, on average, 15.4 times higher than the one of the massive, normal starforming galaxies at Solar luminosity. IZw18 shows even higher $L_{\mathrm{IR}} / L_{\mathrm{CO}(1-0)}^{\prime}$ and $\mathrm{SFR} / L_{\mathrm{CO}(1-0)}^{\prime}$ ratios, which are $\gtrsim 5.7$ and $\gtrsim 119$ times higher than those of other metal-poor galaxies (Fig. 4Top left), respectively. It is difficult for $\mathrm{CO}$ molecules to form in metal-poor environments because there is fewer raw material and little dust to protect the already scarce $\mathrm{CO}$ molecules from UV radiation. The higher ratios are a natural result of this. The $\mathrm{SFR} / L_{\mathrm{CO}(1-0)}^{\prime}$ ratio of metal-poor galaxies increases as the metallicity decreases (Fig. 4-Bottom right). Hunt et al. (2015) found a significant correlation when fitting their dwarf galaxies, detections in Schruba et al. (2012), and the compilation therein. We see a slightly shallower trend when including all galaxies with a $\mathrm{CO}$ detection. We kept only the star-forming galaxies in XCOLD in the regression to exclude the contamination from active galactic nucleis. The SFR/ $L_{\mathrm{CO}(1-0)}^{\prime}$ ratio of IZw18 falls above the trend and it is more than ten times higher than the rest of the galaxies. This suggests a great difference in the $\mathrm{CO}$ distribution and content of galaxies similar to IZw18, at a few percentages of solar metallicity, than that of metal-rich galaxies. However, as we discuss below, the change may occur gradually with the decreased metallicity.

SFR and $L_{\mathrm{CO}(1-0)}^{\prime}$ can be derived from observations, but the molecular gas mass, $M_{\mathrm{H}_{2}}=\alpha_{\mathrm{CO}} \times L_{\mathrm{CO}(1-0)}^{\prime}$, is the key quantity of interest. The observed $\mathrm{SFR} / L_{\mathrm{CO}(1-0)}^{\prime}$ ratio is related to both the depletion time $\left(\tau_{\text {dep }}\right)$ and the CO-to- $\mathrm{H}_{2}$ conversion factor $\left(\alpha_{\mathrm{CO}}\right)$ as $\mathrm{SFR} / L_{\mathrm{CO}(1-0)}^{\prime}=\mathrm{SFR} /\left(M_{\mathrm{H}_{2}} / \alpha_{\mathrm{CO}}\right)=\alpha_{\mathrm{CO}} / \tau_{\text {dep }}$. Many studies have shown that $\alpha_{\mathrm{CO}}$ increases rapidly at low metallicity theoretically and observationally (Israel 1997; Glover \& Mac Low 2011; Leroy et al. 2011; Narayanan et al. 2012; Elmegreen et al. 2013; Shi et al. 2016). If $\tau_{\text {dep }}$ is constant, as is found for the nearby disc galaxies (Leroy et al. 2008; Bigiel et al. 2011), then the dependence of the $\mathrm{SFR} / L_{\mathrm{CO}(1-0)}^{\prime}$ ratio on metallicity is the direct consequence of the variation of $\alpha_{\mathrm{CO}}$. However, $\tau_{\mathrm{dep}}$ in dwarf galaxies remains uncertain and tends to be shorter at a lower mass and at a higher specific SFR (sSFR; Saintonge et al. 2011; Shi et al. 2014; Hunt et al. 2020). IZw18, as a dwarf galaxy with a relatively high sSFR and low mass, is likely to have low $\tau_{\text {dep }}$. We speculates that as $\alpha_{\mathrm{CO}}=\tau_{\text {dep }} \times \frac{\text { SFR }}{L_{\mathrm{CO}(1-0)}^{\prime}}$, the high $\mathrm{SFR} / L_{\mathrm{CO}(1-0)}^{\prime}$ ratio of IZw 18 would overwhelm the possibly low $\tau_{\text {dep }}$, and this would result in a high $\alpha_{\mathrm{CO}}$. Moreover, considering the dependence of $\alpha_{\mathrm{CO}}$ on both sSFR and metallicity for the metal poor galaxies, IZw18 follows the empirical relation found in Hunt et al. (2020) within the uncertainty of the stellar mass, as shown in the bottom right corner of Fig. 5. This relation was derived based on a recent compilation of $\sim 400$ metal poor galaxies (Ginolfi et al. 2020, MAGMA). We note that the individual star-forming regions of the metal-poor galaxies from Shi et al. $(2015,2016)$ all fall slightly above the relation, but well within the scatter of the global measurements of the galaxies. This indicates that $\alpha_{\mathrm{CO}}$ changes continuously with metallicity and sSFR. A further constraint on the conversion factor $\left(\alpha_{\mathrm{CO}}\right)$ of IZw18 is beyond the scope of this paper.

\subsection{The structure of the interstellar medium}

Bolatto et al. (1999) and Röllig et al. (2006) modelled the PDRs and found that as the [C II] regions expand at low metallicity, the $L_{[\mathrm{C} \text { II }} / L_{\mathrm{CO}(1-0)}$ ratio increases. This has also been confirmed in previous observations (Madden 2000; Cormier et al. 2014). Cormier et al. (2015) detected the bright [C II] emission in IZw18. We plotted the $L_{[\mathrm{CII}]} / L_{\mathrm{CO}(1-0)}$ ratio as a function of metallicity and specific SFR in Fig. 5. IZw18 generally follows the trend with metallicity, defined by the galaxies compiled by Zanella et al. (2018, see references therein), but it shows ratios several times higher than the prediction of the regression fit to sSFR. We note that [C II] is considered to be a good tracer of CO-dark molecular gas (e.g. Cormier et al. 2015; Accurso et al. 2017b; Zanella et al. 2018; Madden et al. 2020). Even though [C II] can originate from both ionised gas and neutral gas, simulations and models have found that the ionised fraction does not go beyond 50\%, even for metal-poor galaxies (Accurso et al. 2017a; Cormier et al. 2019). The ionised fraction of IZw18 is unknown, but it could be low as recent studies found a decreasing fraction with decreasing metallicity (Madden et al. 2020, and references therein). This indicates that the total molecular reservoir, traced by [C II] emission from PDRs, is much larger than the CO emitter in IZw18. Then the extreme case of IZw18 reinforces a significant change in the ISM structure in systems at a few percent of the Solar metallicity and this may have similar implications for such systems in the early Universe.

\section{Conclusions}

In this Letter, we report a marginal detection of $\mathrm{CO} J=2-$ 1 in IZw18, using the observation from NOEMA after its Phase II upgrade. We pushed the detection limit of $L_{\mathrm{CO}}^{\prime}$ down to $L_{\mathrm{CO}(2-1)}^{\prime}=3.99 \times 10^{3} \mathrm{~K} \mathrm{~km} \mathrm{~s}^{-1} \mathrm{pc}^{-2}$, which is 40 times lower than that of Leroy et al. (2007). As one of the most metalpoor galaxies, IZw 18 shows $L_{\mathrm{IR}} / L_{\mathrm{CO}(1-0)}^{\prime}$ and $\mathrm{SFR} / L_{\mathrm{CO}(1-0)}^{\prime}$ ratios much higher than those of galaxies with a higher metal abundance. Particularly, the SFR/L $L_{\mathrm{CO}(1-0)}^{\prime}$ ratio of IZw18 constrains the $\mathrm{CO}-$ to- $\mathrm{H}_{2}$ conversion factor to rise considerably at metallicity lower than $5 \% \mathrm{Z}_{\odot}$. The $\mathrm{SFR} / L_{\mathrm{CO}(1-0)}^{\prime}$ ratio also follows the regression found in Hunt et al. (2020) well which considers the influence of both sSFR and metallicity. The high $L_{[\mathrm{C} \mathrm{II}]} / L_{\mathrm{CO}(1-0)}$ ratio indicates that the $\mathrm{CO}$ emitter may trace only the inner part of the entire molecular gas reservoir due to the extremely low 

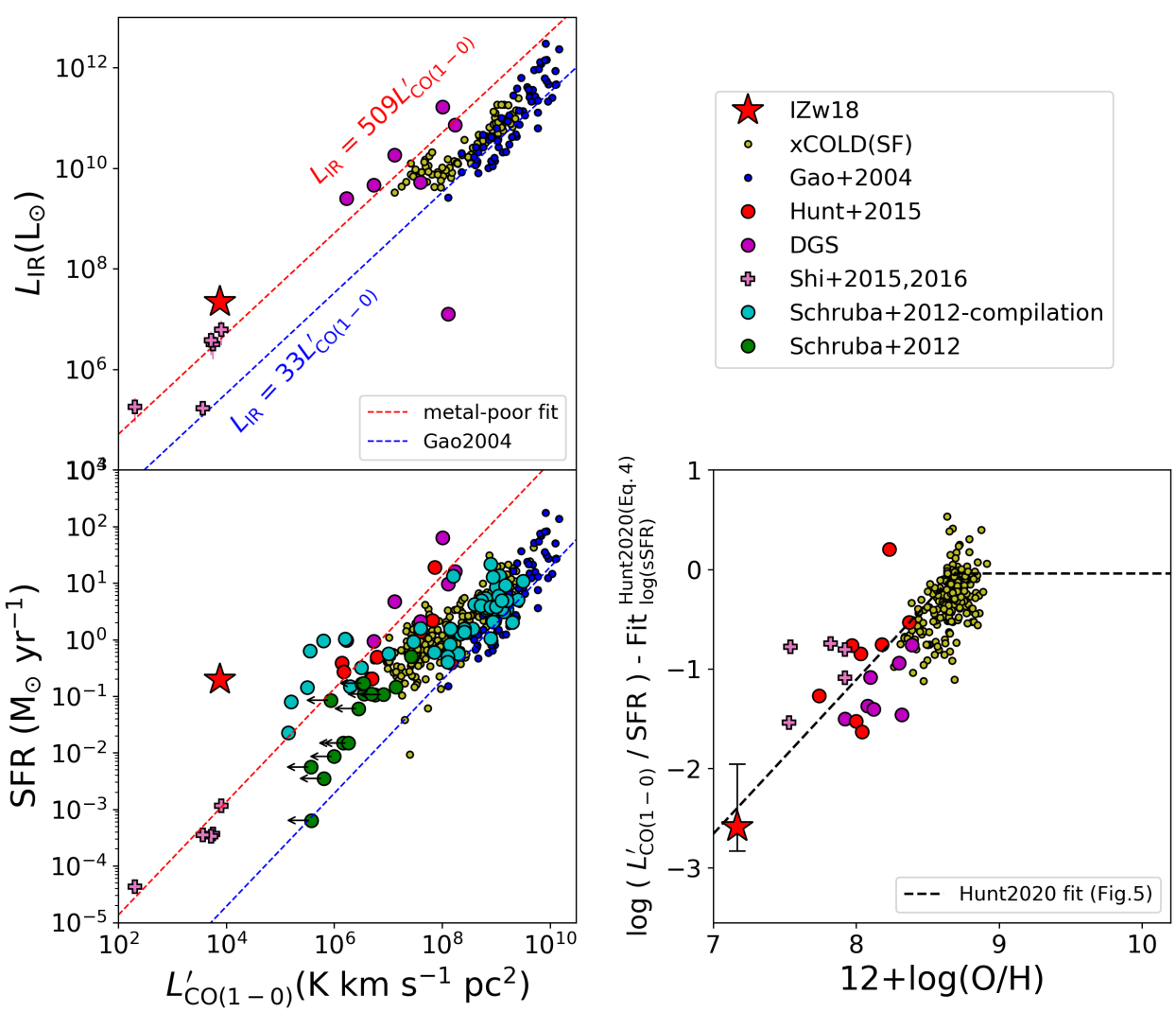

Fig. 4. Infrared luminosity (top left) and SFR (derived from the emission at FUV and $24 \mu \mathrm{m}$, bottom left) versus $L_{\mathrm{CO}(1-0)}^{\prime}$. The upper limit of IZw18 is shown as a red star, in comparison with the galaxies described in Sect. 4. In the two panels on the left, the blue dashed lines show the correlation by Gao \& Solomon (2004) and the red dashed lines show the linear fit of the metal-poor galaxies (Shi et al. 2015, 2016; Hunt et al. 2015; Cormier et al. 2014, DGS). The bottom right panel shows the empirically derived broken power-law regression for $L_{\mathrm{CO}(1-0)}^{\prime} / \mathrm{SFR}$ as a function of metallicity, illustrating the decrease of $\alpha_{\mathrm{CO}}$ with metallicity as introduced by Hunt et al. (2020). The error bar of IZw18 represents the uncertainty in the measurements of the stellar mass. We note that in the panels, all the circles represent the global properties of the galaxies, while the magenta crosses denote the star-forming regions.

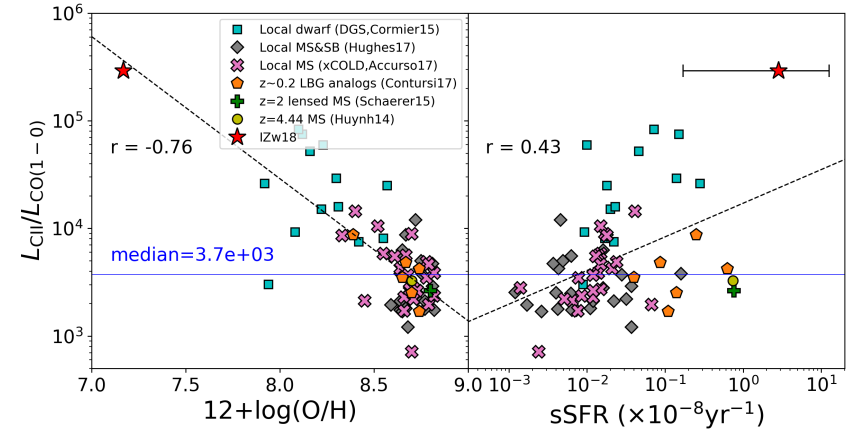

Fig. 5. $L_{\mathrm{CII}} / L_{\mathrm{CO}(1-0)}$ ratio as a function of the metallicity (left) and specific SFR (right). IZw18 (red star) is compared to the galaxies compiled in Zanella et al. (2018, see references therein). The errorbar reflects the uncertainties of stellar mass and SFR. Pearson's correlation coefficients for the regression fits are shown in each panel.

metallicity of IZw18. An upper limit of the continuum emission at $1.3 \mathrm{~mm}$ is also obtained to constrain the Rayleigh Jeans tail of the SED and we excluded a sub-millimetre excess in IZw18.

Acknowledgements. We are grateful to the referee for useful comments which significantly improved the quality of this manuscript. L. Z. and Y. S. acknowledge the support from the National Key R\&D Program of China (No. 2018YFA0404502, No. 2017YFA0402704) and the National Natural Science
Foundation of China (NSFC grants 11825302, 11733002 and 11773013) and the science research grants from the China Manned Space Project with NO. CMSCSST-2021-B02. Y. S. thanks the support by the Tencent Foundation through the XPLORER PRIZE. L. Z. also acknowledges China Scholarship Council (CSC). J. W. is thankful for the support of NSFC (grant 11590783). Z. Y. Z. acknowledges the support of NSFC (grant 12041305) and the Program for Innovative Talents, Entrepreneur in Jiangsu.

\section{References}

Accurso, G., Saintonge, A., Catinella, B., et al. 2017a, MNRAS, 470, 4750 Accurso, G., Saintonge, A., Bisbas, T. G., \& Viti, S. 2017b, MNRAS, 464, 3315

Aloisi, A., Clementini, G., Tosi, M., et al. 2007, ApJ, 667, L151

Asplund, M., Grevesse, N., Sauval, A. J., \& Scott, P. 2009, ARA\&A, 47, 481

Bigiel, F., Leroy, A. K., Walter, F., et al. 2011, ApJ, 730, L13

Bolatto, A. D., Jackson, J. M., \& Ingalls, J. G. 1999, ApJ, 513, 275

Bolatto, A. D., Wolfire, M., \& Leroy, A. K. 2013, ARA\&A, 51, 207

Boquien, M., Burgarella, D., Roehlly, Y., et al. 2019, A\&A, 622, A103

Brown, M. J. I., Moustakas, J., Smith, J. D. T., et al. 2014, ApJS, 212, 18

Capak, P. L., Carilli, C., Jones, G., et al. 2015, Nature, 522, 455

Cormier, D., Madden, S. C., Lebouteiller, V., et al. 2014, A\&A, 564, A121

Cormier, D., Madden, S. C., Lebouteiller, V., et al. 2015, A\&A, 578, A53

Cormier, D., Abel, N. P., Hony, S., et al. 2019, A\&A, 626, A23

Draine, B. T., \& Li, A. 2007, ApJ, 657, 810

Elmegreen, B. G., Rubio, M., Hunter, D. A., et al. 2013, Nature, 495, 487

Elmegreen, B. G., Herrera, C., Rubio, M., et al. 2018, ApJ, 859, L22

Fisher, D. B., Bolatto, A. D., Herrera-Camus, R., et al. 2014, Nature, 505, 186

Fumagalli, M., Krumholz, M. R., \& Hunt, L. K. 2010, ApJ, 722, 919

Galametz, M., Madden, S. C., Galliano, F., et al. 2011, A\&A, 532, A56

Galliano, F., Madden, S. C., Jones, A. P., et al. 2003, A\&A, 407, 159 
Gao, Y., \& Solomon, P. M. 2004, ApJ, 606, 271

Gil de Paz, A., Madore, B. F., \& Pevunova, O. 2003, ApJS, 147, 29

Gil de Paz, A., Boissier, S., Madore, B. F., et al. 2007, ApJS, 173, 185

Ginolfi, M., Hunt, L. K., Tortora, C., Schneider, R., \& Cresci, G. 2020, A\&A, 638, A4

Glover, S. C. O., \& Mac Low, M. M. 2011, MNRAS, 412, 337

Grenier, I. A., Casandjian, J.-M., \& Terrier, R. 2005, Science, 307, 1292

Hunt, L. K., Dyer, K. K., \& Thuan, T. X. 2005, A\&A, 436, 837

Hunt, L. K., Testi, L., Casasola, V., et al. 2014, A\&A, 561, A49

Hunt, L. K., García-Burillo, S., Casasola, V., et al. 2015, A\&A, 583, A114

Hunt, L. K., De Looze, I., Boquien, M., et al. 2019, A\&A, 621, A51

Hunt, L. K., Tortora, C., Ginolfi, M., \& Schneider, R. 2020, A\&A, 643, A180

Israel, F. P. 1997, A\&A, 328, 471

Izotov, Y. I., \& Thuan, T. X. 1999, ApJ, 511, 639

Izotov, Y. I., \& Thuan, T. X. 2004, ApJ, 616, 768

Klein, U., Lisenfeld, U., \& Verley, S. 2018, A\&A, 611, A55

Lelli, F., Verheijen, M., Fraternali, F., \& Sancisi, R. 2012, A\&A, 537, A72

Lelli, F., Verheijen, M., \& Fraternali, F. 2014, MNRAS, 445, 1694

Leroy, A., Cannon, J., Walter, F., Bolatto, A., \& Weiss, A. 2007, ApJ, 663, 990

Leroy, A. K., Walter, F., Brinks, E., et al. 2008, AJ, 136, 2782

Leroy, A. K., Bolatto, A., Gordon, K., et al. 2011, ApJ, 737, 12

Lisenfeld, U., Israel, F. P., Stil, J. M., \& Sievers, A. 2002, A\&A, 382, 860

Madden, S. C. 2000, New Astron. Rev., 44, 249
Madden, S. C., Cormier, D., Hony, S., et al. 2020, A\&A, 643, A141

Narayanan, D., Krumholz, M. R., Ostriker, E. C., \& Hernquist, L. 2012, MNRAS, 421, 3127

Oey, M. S., Herrera, C. N., Silich, S., et al. 2017, ApJ, 849, L1

Rémy-Ruyer, A., Madden, S. C., Galliano, F., et al. 2013, A\&A, 557, A95

Röllig, M., Ossenkopf, V., Jeyakumar, S., Stutzki, J., \& Sternberg, A. 2006, A\&A, 451, 917

Rosolowsky, E., Leroy, A. K., Usero, A., et al. 2015, Am. Astron. Soc. Meeting Abstr., 225, 141.25

Rubio, M., Elmegreen, B. G., Hunter, D. A., et al. 2015, Nature, 525, 218

Saintonge, A., Kauffmann, G., Wang, J., et al. 2011, MNRAS, 415, 61

Saintonge, A., Catinella, B., Tacconi, L. J., et al. 2017, ApJS, 233, 22

Schruba, A., Leroy, A. K., Walter, F., et al. 2012, AJ, 143, 138

Schruba, A., Leroy, A. K., Kruijssen, J. M. D., et al. 2017, ApJ, 835, 278

Shi, Y., Armus, L., Helou, G., et al. 2014, Nature, 514, 335

Shi, Y., Wang, J., Zhang, Z.-Y., et al. 2015, ApJ, 804, L11

Shi, Y., Wang, J., Zhang, Z.-Y., et al. 2016, Nat. Commun., 7, 13789

Shi, Y., Wang, J., Zhang, Z.-Y., et al. 2020, ApJ, 892, 147

Solomon, P. M., \& Vanden Bout, P. A. 2005, ARA\&A, 43, 677

Walter, F., Decarli, R., Carilli, C., et al. 2012, ApJ, 752, 93

Warren, S. R., Molter, E., Cannon, J. M., et al. 2015, ApJ, 814, 30

Wolfire, M. G., Hollenbach, D., \& McKee, C. F. 2010, ApJ, 716, 1191

Zanella, A., Daddi, E., Magdis, G., et al. 2018, MNRAS, 481, 1976

\section{Appendix A: Multiwavelength images}

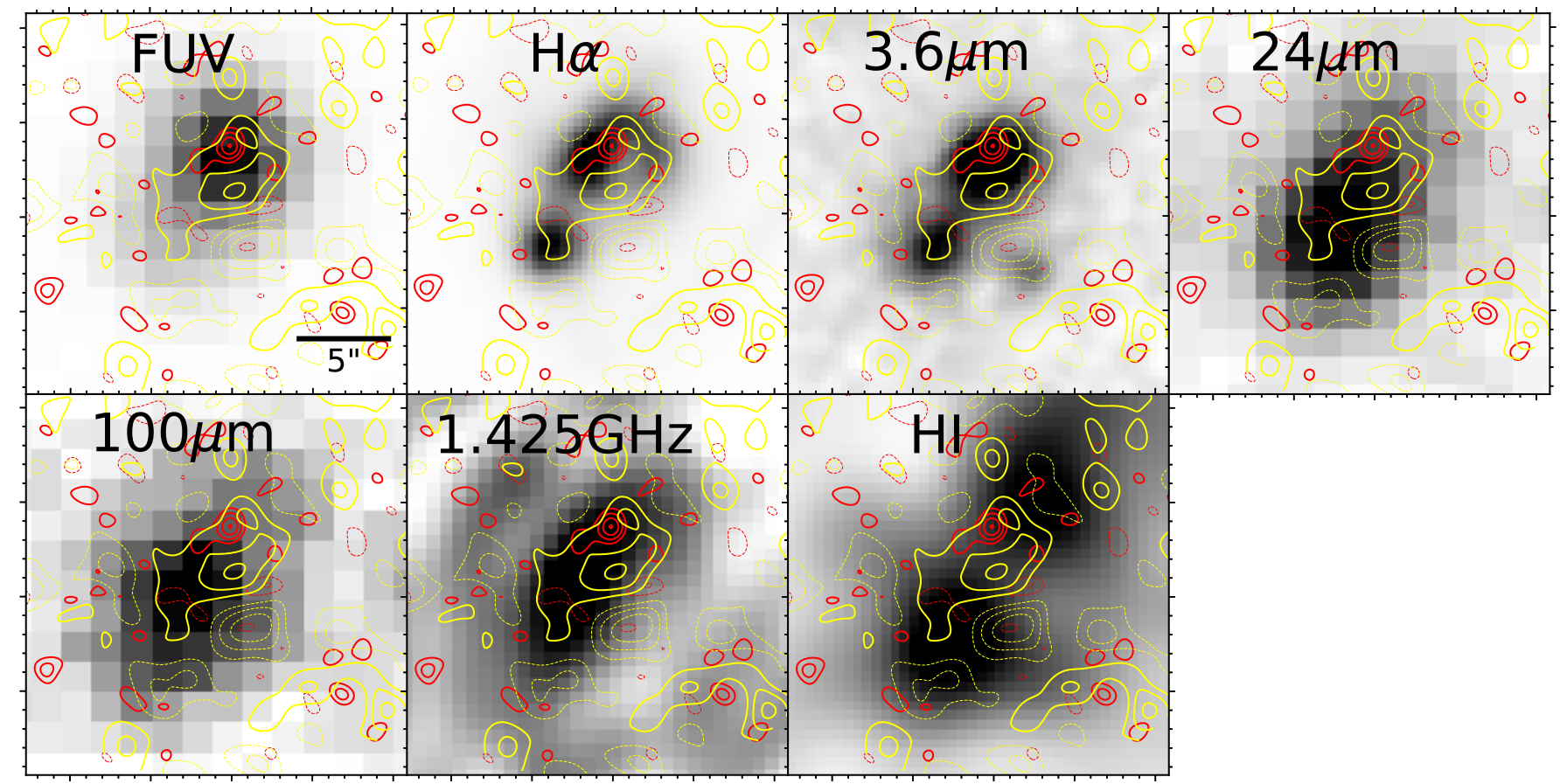

Fig. A.1. Similar to Fig. 1, but CO $J=2-1$ emission and $1.3 \mathrm{~mm}$ continuum superimposed on the GALEX FUV (Gil de Paz et al. 2007), H $\alpha$ (Gil de Paz et al. 2003), Spitzer IRAC1 at $3.6 \mu \mathrm{m}$ (Brown et al. 2014), Spitzer MIPS $24 \mu \mathrm{m}$ (Brown et al. 2014), Herschel MIPS at 100 $\mu \mathrm{m}$ (Fisher et al. 2014), and VLA $L$-band at $1.425 \mathrm{GHz}$ (Hunt et al. 2005) continuum maps, as well as the H I gas intensity map (Lelli et al. 2012). The $\mathrm{CO}$ emission coincides with emissions at $\mathrm{FUV}, \mathrm{H} \alpha$, and radio continuum, which trace star formation, along with stellar emission at $3.6 \mu \mathrm{m}$. Meanwhile, the hot and cold dust emission at $24 \mu \mathrm{m}$ and $100 \mu \mathrm{m}$ show a slight offset $\left(\sim 3^{\prime \prime}\right)$ from the CO emission. The extended emission at $1.3 \mathrm{~mm}$ generally covers the emission at all bands shown here, except for $\mathrm{HI}$ gas. Emission at the $1.3 \mathrm{~mm}$ continuum falls between the two peaks of $\mathrm{H}$ I gas emission, and CO emission is also shifted from one of the $\mathrm{HI}$ peaks by $\sim 2$ ". A clear offset is shown between $\mathrm{HI}$ gas and CO gas and the $1.3 \mathrm{~mm}$ continuum. An offset between CO gas and H I gas has also been observed in another metal-poor galaxy, Sextans B (Shi et al. 2016). 